\section{A fatal case of acute bentazone overdose despite cricothyroidotomy during cardiopulmonary resuscitation}

\section{Kyungwon Lee}

Department of Emergency Medicine, Inje University Seoul Paik Hospital, Seoul, Korea

Bentazone is classified as a moderately hazardous (class II) herbicide by the World Health Organization. A 53-year-old Korean woman was transferred to the emergency department after a suicide attempt using approximately $500 \mathrm{~mL}$ of bentazone one hour prior to admission. Upon admission, she was alert and tachycardia of 125/min was observed. She was treated with gastric lavage and activated charcoal, during which she experienced diarrhea. Two hours after bentazone ingestion, cardiac arrest and muscle rigidity throughout the body occurred. Cardiopulmonary resuscitation was immediately started. Endotracheal intubation after administration of a muscle relaxant (succinylcholine) was unsuccessful because of temporomandibular joint muscle rigidity. Surgical cricothyroidotomy was performed by the emergency physician, but the patient was not resuscitated. For cardiac arrest patients with muscle rigidity caused by bentazone overdose, endotracheal intubation may be challenging because of muscle rigidity, despite appropriate use of muscle relaxants. Early surgical cricothyroidotomy may be the preferred method of airway management in these patients.

Keywords Bentazone; Drug overdose; Fatal outcome

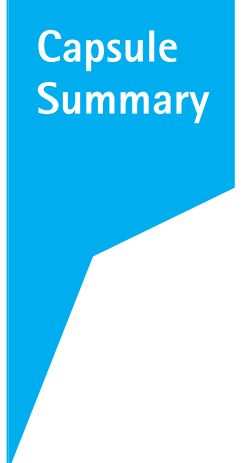

What is already known

Acute bentazone overdose is uncommon, and the symptoms of acute bentazone overdose are gastrointestinal, respiratory, cardiovascular, and musculoskeletal (such as muscle rigidity).

What is new in the current study

We found that the depolarizing skeletal muscle relaxant succinylcholine might not be able to resolve muscle rigidity caused by acute bentazone overdose. Early surgical cricothyroidotomy may be the preferred method of airway management in these patients.
eISSN: 2383-4625

Received: 21 May 2017

Revised: 20 July 2017

Accepted: 21 July 2017

Correspondence to: Kyungwon Lee Department of Emergency Medicine, Inje University Seoul Paik Hospital, 9 Mareunnae-ro, Jung-gu, Seoul 04551, Korea

E-mail:emkwlee@gmail.com

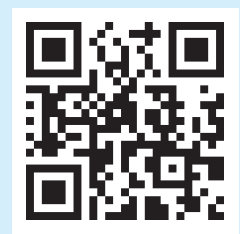

How to cite this article:

Lee $K$. A fatal case of acute bentazone overdose despite cricothyroidotomy during cardiopulmonary resuscitation. Clin Exp Emerg Med 2017;4(4):254-257.

This is an Open Access article distributed under the terms of the Creative Commons Attribution Non-Commercial License (http:// creativecommons.org/licenses/by-nc/4.0/). 


\section{INTRODUCTION}

Intentional ingestion of agricultural pesticides and herbicides for suicide accounts for most of the cases of acute overdose in hospital emergency departments (EDs) in Korea. ${ }^{1}$ Such cases differ in toxicological epidemiology from those reported in other developed countries. The World Health Organization classifies bentazone as a moderately hazardous (class II) herbicide. ${ }^{2}$ Although fatal cases of acute bentazone overdose have been reported in other countries, ${ }^{3-6}$ a surgical airway such as cricothyroidotomy during cardiopulmonary resuscitation (CPR) for the victim has not yet been reported. We report a fatal case of acute bentazone overdose despite cricothyroidotomy during CPR.

\section{CASE REPORT}

A 53-year-old Korean woman was brought to the ED of a community hospital that had 250 in-patient beds by the 119 emergency medical services ambulance crew owing to pesticide poisoning. The 119 is a universal telephone code for fire, emergency medical services, and disaster in Korea. About an hour earlier, the patient attempted suicide at home by ingesting an herbicide. Upon hospital admission, the patient was alert, with a Glasgow Coma Scale score of 15, and the following vital signs: blood pressure of $141 / 78 \mathrm{mmHg}$, pulse rate of $125 / \mathrm{min}$, respiratory rate of $18 / \mathrm{min}$, body temperature of $36^{\circ} \mathrm{C}$, and oxygen saturation of $97 \%$. The trade name of the herbicide was Basagran, a herbicide comprising bentazone (40\%) and a solvent (60\%). The $500-\mathrm{mL}$ vessel was empty. Upon the patient's arrival, $1 \mathrm{~L}$ of normal saline was administered intravenously into her right basilic vein, emergency blood sampling was conducted, and gastric lavage with a large bore orogastric tube was performed with $4 \mathrm{~L}$ saline solution. The lavage cleared the gastric contents; they were bright red in color. About 10 minutes after the patient's arrival to the hospital, during the gastric lavage, the patient defecated with loose stools indicative of diarrhea. About 25 minutes after the patient's arrival at the ED, a 16-Fr nasogastric tube was inserted, through which the emergency physician administered $50 \mathrm{~g}$ of activated charcoal. Forty minutes after the patient's arrival at the ED, the patient's complete blood count and urine analysis results were reported. They were normal. The emergency physician continuously monitored the patient at the bedside, and nurses prepared to transfer the patient to the intensive care unit and removed the ECG lead and oxygen saturation monitoring probe of the fixed monitor. About 52 minutes after the patient's arrival at the ED, the emergency physician noticed that her consciousness suddenly deteriorated while her breathing weakened. Immediately, the nurses re- attached the ECG lead and oxygen saturation probe, and the emergency physician identified the ECG rhythm as asystolic. The oxygen saturation could not be determined. Immediately, chest compression and bag-mask ventilation were performed, and 1 $\mathrm{mg}$ of epinephrine was administered intravenously through the peripheral vein. Since then, $1 \mathrm{mg}$ of epinephrine was injected intravenously every 2 to 3 minutes. Oxygen was administered at a flow rate of $15 \mathrm{~L} / \mathrm{min}$ through a bag mask. After 2 minutes of $\mathrm{CPR}, 20 \mathrm{mg}$ of etomidate was injected intravenously for endotracheal intubation. However, the temporomandibular joint remained firmly fixed. The muscles of the body, including those of the extremities, were rigid. After 3 minutes of CPR, a muscle relaxant, succinylcholine (50 mg), was injected intravenously. After succinylcholine injection, the temporomandibular joint did not show any relaxation response. Physical traction of the joint was ineffective. After 7 minutes of CPR, $100 \mathrm{mg}$ of succinylcholine was injected intravenously, but the temporomandibular joint did not relax. While the chest compressions and bag mask ventilation continued, and the ECG remained asystolic, the emergency physician performed needle-cricothyroidotomy. Finally, the emergency physician successfully performed surgical cricothyroidotomy after 12 minutes of CPR. After surgical cricothyroidotomy, the oxygen saturation was 100\%, but the ECG continuously showed asystole. The emergency physician declared the victim dead after $63 \mathrm{~min}$ utes of CPR. A total of $21 \mathrm{mg}$ of epinephrine had been administered. The body, including the articular jaw, fingers, and toes, was stiff and showed no change at all until death. During CPR, the initial blood biochemical test results were reported as normal, except for the $\mathrm{Na}^{+}$result, which was 148 (136 to 145) mmol/L. No autopsy or toxicological study was performed.

\section{DISCUSSION}

Bentazone is a herbicide that inhibits carbon dioxide fixation in plants such as rice and corn, thus interfering with photosynthesis. ${ }^{5,6}$ In a rat model, bentazone was rapidly absorbed and mostly excreted in the urine. ${ }^{7}$ An hour after its oral administration, it was distributed to the stomach, liver, kidneys, and heart of the rat, but not to its brain or spinal cord.? The substance was metabolized to $6-\mathrm{OH}$ bentazone and $8-\mathrm{OH}$ bentazone through hydroxylation. The symptoms of acute bentazone overdose in the experimental animal model were shallow accelerated breathing, dyspnea, central nervous system depression, pronounced increase in body temperature, and rapid onset of rigor mortis. ${ }^{8}$ The lethal dose (LD 50) was $1,100 \mathrm{mg} / \mathrm{kg}$ in rats and $2,918 \mathrm{mg} / \mathrm{kg}$ in pheasants. ${ }^{6}$ Acute intoxication did not cause any statistically significant changes in the hemoglobin, erythrocyte, or leukocyte counts. ${ }^{6}$ 
Turcant et al. ${ }^{5}$ noted that the effects of benzodiazepines or competitive neuromuscular blocking agents and the mechanism of the muscle rigidity caused by bentazone are yet to be evaluated. Direct muscular toxicity was suspected, but central nervous system toxicity was very unlikely because in the experimental animal model, bentazone did not cross the blood-brain barrier.

Bentazone may also have direct toxicity on the kidney and liver. ${ }^{6}$ Wu et al. ${ }^{6}$ proposed that direct organ toxicity in acute bentazone overdose may be because of the chemical nature of bentazone metabolism. Through bentazone metabolism, $\mathrm{OH}$ metabolites, oxidative stress, and oxygen free radicals were produced. These reactive oxygen species can induce oxidative modification of cellular carbohydrates, proteins, lipids, and nucleic acids, ${ }^{9}$ especially in organs with high metabolic rates and oxygen demand such as muscle, liver, and kidneys. ${ }^{6}$ As mentioned before, in the animal model, bentazone was rapidly absorbed in and distributed to the stomach, liver, kidneys, and heart. We thus speculated that bentazone can be rapidly distributed to and accumulate in specific organs such as the muscles, including the heart, gastrointestinal tract, respiratory system, and skeletal muscles, and can directly damage them and eventually cause organ failure. Muller et al. ${ }^{4}$ proposed that a dose of 686 to 1,371 $\mathrm{mg}$ ingested per $\mathrm{kg}$ of body weight is within the predictable lethal ingestion range.

Acute bentazone overdose is uncommon. We searched Medline for cases of acute bentazone overdose from 1966 to 2015, and found only four English papers on human acute bentazone over- dose, including three fatal cases in France, Denmark, and China ${ }^{3-6}$ (Table 1). The symptoms of acute bentazone overdose were gastrointestinal, such as nausea, vomiting, diarrhea, and abdominal pain, respiratory, such as cough, tachypnea, dyspnea, and apnea, cardiovascular, such as tachycardia and cardiac arrest, and musculoskeletal, such as muscle rigidity. The initial symptoms were gastrointestinal, such as nausea, vomiting, and diarrhea. In the cases of acute bentazone overdose, acute renal failure and acute toxic hepatitis developed. In all the reported cases, the overdose route was oral, the reason for the overdose was a suicide attempt, and the victims were male. In our case, the symptoms were similar to those of the other reported cases: tachycardia, diarrhea, muscle rigidity, and cardiac arrest. The route and the reason for the overdose were the same as those in the previously reported cases. The difference between the previously reported cases and our case is that in the previous reports, the physicians did not attempt any form of surgical airway procedure, while in our case, the emergency physician performed surgical cricothyroidotomy. However, even though surgical airway insertion was performed in our case, the mortality outcome was the same.

Through our case, we found that the depolarizing skeletal muscle relaxant succinylcholine might not be able to reverse the muscle rigidity caused by acute bentazone overdose. Development of muscle rigidity was observed before injection of succinylcholine, and even after three times the normal $1 \mathrm{mg} / \mathrm{kg}$ dose of succinylcholine was used, no relaxation of the rigid muscles was observed.

Table 1. Comparison of demographic and clinical parameters of this case with those reported in the published studies of acute bentazone overdose

\begin{tabular}{|c|c|c|c|c|}
\hline Variable & Case A (current case) & Case $B^{4}$ & Case $\mathrm{C}^{5}$ & Case $D^{6}$ \\
\hline Age/sex & 53/female & 59/female & $56 /$ male & $31 / \mathrm{male}$ \\
\hline Country & Korea & Denmark & France & China \\
\hline Composition & Bentazone (40\%) & Bentazone (48\%) & Bentazone $(48 \%)$ & Bentazone (44\%) \\
\hline Dose (mL) & 500 & $100-200$ & 500 & 200 \\
\hline Reason & Suicide & Suicide & Suicide & Suicide \\
\hline Route & Oral & Oral & Oral & Oral \\
\hline Coingestants & None & Alchohol, citalopram & Citalopram & None \\
\hline Prehospital time & $1 \mathrm{hr}$ & $<12 \mathrm{hr}$ & $1 \mathrm{hr}$ & 3 day \\
\hline Initial manifestation & Diarrhea & Vomiting, diarrhea & Vomiting, diarrhea & Nausea, vomiting \\
\hline Respiratory symptom & Repiratory failure & Dyspnea & Polypnea, apnea & Respiratory failure \\
\hline Cardiovascular & Tachycardia, cardiac arrest & Cardiac arrest & Cardiac arrest & Tachycardia \\
\hline CNS & Agitation & Drowsy with muddled speech & Agitated, sweating & No \\
\hline Musculoskeletal & Muscle rigidity & No & Muscle rigidity & No \\
\hline Others & & Abdominal pain & & Acute hepatitis, ARF \\
\hline Management & CPR & ED resuscitation attempt failed & CPR & $\begin{array}{l}\text { Hydration, dialysis, CPR were not } \\
\text { performed due to family refusal }\end{array}$ \\
\hline Endotracheal intubation & Failed & & Failed & \\
\hline Surgical cricothyroidotomy & Done & & Not tried & \\
\hline Hospital day & $2 \mathrm{hr}$ & 2 day & $2 \mathrm{hr}$ & 3 day \\
\hline Outcome & Died & Died & Died & Died \\
\hline
\end{tabular}

CNS, central nervous system; ARF, acute renal failure; CPR, cardiopulmonary resuscitation; ED, emergency department. 
To our knowledge, this is the first reported fatal case of acute bentazone overdose, despite cricothyroidotomy during CPR. The muscular toxicity seen in acute bentazone overdose has already been reported in previous fatal cases, ${ }^{5}$ but no attempts have been made to perform a surgical airway procedure during CPR. In this case, the emergency physician performed surgical cricothyroidotomy after failure of rapid-sequence intubation. In addition, we found that the muscle relaxant succinylcholine might be ineffective against muscle rigidity in acute bentazone overdose.

\section{CONFLICT OF INTEREST}

No potential conflict of interest relevant to this article was reported.

\section{REFERENCES}

1. Sung AJ, Lee KW, So BH, et al. Multicenter survey of intoxication cases in Korean emergency departments: 2nd annual report, 2009. J Korean Soc Clin Toxicol 2012;10:22-32.

2. World Health Organization. The WHO recommended classification of pesticides by hazard and guidelines to classification 2009 [Internet]. Geneva: World Health Organization; 2010 [cited 2015 May 20]. Available from: http://www.who.int/ipcs/ publications/pesticides_hazard/en/.

3. Lin TJ, Hung DZ, Hu WH, Yang DY, Wu TC. Acute basagran poisoning mimicking neuroleptic malignant syndrome. Hum Exp Toxicol 1999;18:493-4.

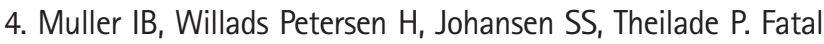
overdose of the herbicide bentazone. Forensic Sci Int 2003; 135:235-6.

5. Turcant $A$, Harry $P$, Cailleux $A$, et al. Fatal acute poisoning by bentazon. J Anal Toxicol 2003;27:113-7.

6. Wu IW, Wu MS, Lin JL. Acute renal failure induced by bentazone: 2 case reports and a comprehensive review. J Nephrol 2008;21:256-60.

7. Chasseaud LF, Hawkins DR, Cameron BD, Fry BJ, Saggers VH. The metabolic fate of bentazon in the rat. Xenobiotica 1972; 2:269-76.

8. Neuschl J, Kacmar P. Acute oral toxicity of bentazon, an herbicide developed in Czechoslovakia, in pheasants and rabbits and the clinical symptoms of poisoning. Vet Med (Praha) 1993; 38:115-21.

9. Huber R, Otto $S$. Environmental behavior of bentazon herbicide. Rev Environ Contam Toxicol 1994;137:111-34. 